\title{
Schools as social and learning communities: Scotland and Norway
}

Terry Wrigley,

with Nina Fjeld,

University of Edinburgh

\begin{abstract}
This article reports on a pilot study examining the possible impact of the very different structures of Scottish and Norwegian schools. It compares the situation in Scotland, as in other parts of the UK, where lower secondary classes normally have 12-15 teachers, each for 1-3 hours, and the Norwegian school, where a team of 5 or 6 teachers is attached to a year group, providing for the pupils' ${ }^{i}$ personal needs and social development as well as the academic curriculum. It seeks to relate these different structures to a greater level of trust and connectedness between Norwegian pupils and teachers, as well as the pupils' sense of personal efficacy.
\end{abstract}

This exploratory study points towards a relationship between pupil well-being and school size and structure. This is set against the background of

a) the high frequency of exclusions from the lower years of Scottish secondary schools, particularly among boys of lower socio-economic backgrounds; and

b) research which shows that pupils develop less troubled behaviour and enjoy better relationships in smaller schools, and that ethnic minority and low SES pupils in particular achieve higher.

The former is indicative of considerable turbulence in the early years of Scottish secondary schools; the latter points towards a probable relationship between school size and structure, on the one hand, and ethos and achievement on the other, particularly for less advantaged populations. Whilst falling short of a 'proof', this small pilot study, conducted with limited resources, nevertheless provides sufficient data to suggest that we should critically reexamine some of the current norms of secondary school organisation in the UK and similar systems. The study has implications for the generation of social capital and the development of learning communities.

Keywords: Structure; school size; learning community; social capital; exclusion; transition

\section{School structure and ethos in Scotland and Norway}

The organisation of schools, particularly in the lower secondary years, has been a longstanding if sporadic interest. Many years ago, the work of Maurice Galton and others (e.g. Galton and Willcocks 1983) focused on the difficulties of transfer, and clearly demonstrated a dip in progress and achievement following transition from primary to secondary school. I had worked in two secondary schools in England which had successfully created greater cohesion between a year group of pupils and a team of teachers. In the second of these schools (early-mid 1980s), a six-form entry 11-16 school, first year pupils had spent about $40 \%$ of their time with a class teacher, including form time / PSE, English, maths, history and geography. For the rest of the school, although the timetabling was more tradition, the subject department was also the pastoral team for a year group, with the same suite of rooms serving as the department's teaching space and the year group's social space. This served very well in most cases, though depending on sufficient commitment from each team of teachers. This suggests that a different structure can provide conditions to support a cultural change, but does not automatically lead to that result. 
The Scottish schools inspectorate, in Achieving Success in S1/S2 (SOEID, 1997:11), highlighted the difficulties in the lower secondary school:

Most pupils are taught by a greater number of teachers in S1/S2 than at any other stage in their education. It is not unusual for S1 pupils to be taught by between 13 and 16 different teachers each week. In some cases the number rises to 20 or more. Although the variety of the range of contacts can be popular with pupils, limiting the number of their teachers can make it easier for them to adapt to a new school and to learning effectively. Frequent changes of class throughout the day can lead to a loss of time when settling into a lesson or preparing to move on to the next class. The necessity for tasks to be completed within a single period may not allow sufficient time for lessons which combine direct teaching with genuinely investigative or openended learning activities. Taking account of prior learning and monitoring pupils' progress adequately can be very difficult when periods are short and relatively infrequent.

The inspectors' survey showed the longest contact between a class and a particular teacher (English or maths) to be around 12-13\% of the week (less than three hours a week), and in the case of religious education or music only $4-6 \%$.

In contrast with the clarity of the diagnosis, the recommended treatment was half-hearted. Organisationally, it amounted to a proposal to remove some subjects (basically, non-statutory ones designed to provide an introduction and selling-point for later options) and to use some rotational timetabling (e.g. between different arts or humanities). The target was to reduce the number of teachers a class would face to 10-12 in total. This seemed likely to have only a limited impact on cohesion and relationships. It did not make the necessary structural change to support implementation of its other recommendations (e.g. that teachers should pay more attention to progression and meeting individual needs). Moreover, it would clearly not overcome a key problem of coherence - that of developing better teamwork between the teachers responsible for a particular class. This is exacerbated by the common timetabling practice of allocating teachers randomly to lower secondary classes according to who is left over after timetabling the older years. It is rarely feasible for the ten or twelve teachers who happen to be teaching 1B to meet regularly to discuss emerging issues and individual problems.

Working as an inspector, I had frequently been aware of the gulf between intention and reality, in terms of meeting the needs of individual pupils. The care taken by learning support staff, for instance, in assembling and transmitting academic and pastoral details of individual pupils was often wasted, as the many different teachers who each taught the pupil for one, two, at the most three hours a week were simply overloaded with information, and often struggled to learn pupils' names. The same learning support teachers, and those with pastoral and management roles, would then spend much of their time dealing with incidents that resulted from clashes between subject specialists and children whom they did not know very well. Increasingly, bureaucratic answers have been sought for a problem which was structurally rooted, i.e. the lack of sustained contact time between a class and its teachers.

Galton's research was partly replicated twenty years later. In the intervening period, numerous practices had been introduced including meetings between members of staff of the primary and secondary schools, induction days, open evenings, joint projects, summer schools and teacher exchanges. (A full list appears in Galton, Gray and Rudduck 1999:24.) Despite all this, and greater continuity as a result of National Curriculum assessments, the emotional and cognitive problems were still substantial. Nearly $40 \%$ of pupils made no progress or regressed, in absolute terms, during the first year of secondary school, compared with their attainment, using the same test, at the end of primary school (ibid: 9-10). Problems of engagement with school (social isolation, lack of enjoyment, low motivation) persisted even at the end of the first year of the new school, with some surprising findings. (Whereas isolation and motivation problems were greater for the lower attainers, the higher attainers 
were more likely to experience diminished enjoyment, including liking their teachers less and being less happy at what they were told to do.) (Galton, Comber and Pell 2002: 138-9) Delamont and Galton (1986:91) devote a chapter to the 'Luton Airport Syndrome', a term coined by David Hargreaves to describe the homelessness of pupils who pack into crowded corridors to move from room to room every forty minutes, trailing all their belongings with them. It is surprising then that Galton and his various colleagues do not really pursue the possible relationship between secondary school structure and the problems of school tranfer.

Some alternative patterns can be found in various education systems in Europe and America. In the United States, a reform movement known as the Coalition of Essential Schools works on the principle that no teacher should have contact with more than 80 pupils altogether, in contrast to the 200-500 pupils under the typical British school structure. The greater length of time with each class was designed to enable the teachers to develop in-depth learning rather than a rush to cover curriculum content (www.essentialschools.org). The successful adoption of a 'class teacher' pattern in the first year of secondary in an English school, whereby a single teacher has the pastoral responsibility and teaches English and humanities subjects amounting to around 40\% of the timetable, is described in Wrigley (2000:90-2), and other patterns are summarised in Wrigley (2003: 143-5). Various patterns are summarised in the small high schools webpages of the Bill and Melinda Gates Foundation (www.gatesfoundation.org/Education/SmallHighSchools). A reform is underway in the Netherland, were the policy of significantly reducing the number of different teachers per class now has all-party support (Sissing and Weeber 2001; 2002). In some Scandinavian countries, teachers are qualified to teach several different specialisms, so that each class has only four or five teachers altogether. In 2003, a group of Norwegian headteachers visited some Scottish schools. We subsequently invited one of them to speak to a conference of Scottish headteachers. She declared, to stunned silence, 'I just don't understand how you manage to work in your secondary schools. I think they are virtually unmanageable.'

\section{The structure of Scottish and Norwegian schools}

Publicly funded secondary schools in Scotland are all organised on comprehensive principles. With few exceptions, they consist of six school years (12-18), with school attendance being compulsory to age 16 . Each subject is taught by a qualified specialist, and most secondary teachers' professional identities are strongly grounded in a sense of subject specialism. The normal pattern of teacher qualification is at least two years undergraduate study in that subject, a PGCE centred on the subject methodology, and a subject-specific registration with the General Teacher Council for Scotland (GTCS). Until recently, when broader faculty structures have been introduced in some education authorities, the line management was to a Principal Teacher (usually one for each subject, including separate sciences). In many larger schools, the strength of the subject allegiance is further marked and supported by teachers taking coffee and lunchbreaks within their own department, and meeting other colleagues less frequently.

This is balanced, at school level, by guidance, management, and learning support structures ${ }^{\mathrm{ii}}$. The first two align primarily with age cohorts, so that a guidance teacher has responsibility for a year group and a senior manager, in addition to specific whole-school issues, for two year groups.

This structure is often assumed to function well, but it does not make communications straightforward; where it functions less well, it tends to produce a 'balkanised' culture (Hargreaves 1994). One metaphor would be to see the subject departments as constituting the nave of a church, with a tendency for cracks to appear. It is the function of the guidance, learning support and management teams to hold the building together. Although recently there have been particular attempts to encourage teachers to view their role more holistically, this has been resisted by those who see their role as providing instruction in the subject while the guidance teachers deal with adolescent idiosyncrasies. 
The Norwegian pattern is also based on comprehensive principles, but the ages of transfer are different. The lower secondary phase (the 'youth school') begins slightly later, around the age of 13 compared with 12 in Scotland, and lasts for three school years, until age 16. (In some regions, this stage takes place within the same school as primary education, i.e. in the 'children's school', without a transfer.) From age 16-19, the upper secondary schools (the 'continuing schools') each provide a general academic track (pre-university) and at least one vocational specialism. Norwegian schools are generally small, in comparison with Scottish schools, with 80-100 pupils per school year being standard even in urban areas, and often far fewer in more remote rural areas.

Unlike Scottish (and other British) secondary schools, the Norwegian 'youth school' is organised in year groups, which have both an academic and social function. A year group of 100 pupils typically has a team of 5 or 6 teachers, who between them teach the whole curriculum, take care of pupils' welfare and social development, liaise with parents, and provide academic and personal guidance. The team generally includes a teacher with particular interests and qualifications in learning support. The team meeting has the power to take decisions on timetable changes, so that it is easy to organise cross-curricular projects, residentials, and so on. In schools of this size, teachers do not normally teach outside of the particular year group in any one academic year, though a music specialist, for example, might be borrowed by another year team which lacks that skill. Some may move up with the cohort at the end of the year. Sometimes a teacher is stretched too far beyond their qualifications, however; we encountered one teacher who was being expected to teach six different subjects, and felt it a strain.

The strength of subject identity is nowhere near as strong as among Scottish teachers. Even in the first year of the 16-19 school, where there is a core curriculum in the general academic branch, it is common for more than one subject to be taught by the same teacher. Thus, one teacher might teach Norwegian and English or history, whilst another teaches 'Realfag' (i.e. sciences with mathematics, conceived as a single subject).

This pilot study was designed to investigate some possible implications of this key structural difference between the two systems. It is clearly impossible to separate out issues of overall school size from those of internal structure when exploring issues such as engagement and relationships. Also, inevitably, other factors are at work within the respective traditions of the two countries. Nevertheless, at an exploratory level, the study should be valuable in beginning to identify ways in which school structures may provide conditions which foster a particular social and learning culture.

\section{Existing literature on school size and structure}

A new pedagogical paradigm, which we might loosely term 'situated learning', and deriving from Vygotskian social constructivism, implies a need to develop a more contextualised understanding of learning processes and learning cultures. (Hughes 2004; Osborn et al 2003:225) This pilot study can be seen, in part, as contributing to a more contextualised understanding.

The subject-specialist structure of British secondary schools is so nearly universal ${ }^{i i i}$ that there are no systematic studies to compare different approaches. There are occasional case studies (including my own, Wrigley 2000:54 and 90-92) which examine variations. There are a number of American studies based on major school reforms such as the Coalition of Essential Schools (www.essentialschools.org) and diverse models of small school reforms currently sponsored by the Gates Foundation (www.gatesfoundation.org/education/TransformingHighSchools)

The issue of school size, particularly in more deprived urban areas, has been a widespread concern in the USA over the last 10-15 years. Major reforms are now underway in a number of large cities, including New York, Chicago and Boston. The Gates Foundation has given substantial support to this reform movement, including a variety of different models which 
usually involve re-structuring to improve learning and social cohesion as well as a reduction in pupil numbers. Research on school size has ranged from large-scale quantitative studies, sometimes involving thousands of schools across several states, to more specific qualitative investigations of case studies, including schools in the process of sub-division or replacement by smaller schools. The studies have sought to correlate size with achievement and with social outcomes, in addition to issues such as teacher collaboration and job satisfaction.

Some of this research is summarised by Flecknoe (2003) in a previous issue of this journal, and by Kahne et al (2005) in the current issue. In the USA Kathleen Cotton's metastudy (1996, and recently updated electronic version) references 103 previous studies, covering a mixture of outcomes. Its findings point to the superiority of smaller schools in terms of:

- more positive student attitudes

- better social behaviour (measured by truancy, discipline problems, violence, theft, substance abuse and gang participation)

- higher levels of school attendance

- a smaller dropout

- a greater sense of belonging

- higher student academic and general self-concepts

- more positive interpersonal relations between and among students, teachers and administrators

- teacher attitudes towards their work.

Cotton concludes that half the studies which consider achievement show no difference between smaller and larger schools, the other half find smaller schools achieving superior results, but no study suggests that larger schools achieve better results.

A recent government-sponsored English metastudy (Garrett 2004) agrees fully on the social aspects, but is more sceptical on academic success, finding a variable pattern. In particular, the only four UK studies examined (all from England, and three of them using an identical dataset) show attainment improving up to around 900 pupils (11-16 schools; 300 higher for 11-18 schools) but then flattening and peaking at around 1200 before gradually declining. Garrett is unable to establish why the pattern in England appears different from the USA. It is conceivable that this results from the way the market system operates in England, where more popular schools are encouraged to expand up to (and sometimes beyond) their physical capacity, leaving less popular schools with many empty places, and subsequently compelled to accept pupils expelled from the more popular schools. Moreover, the additional pupils attracted to the growing schools tend to be children of more affluent or knowledgeable or determined parents. Thus, in the English context, achievement differences leads to a change in size, rather than simply size affecting achievement.

It is worth noting, however, that even in this environment, the correlation of attainment with size, though 'statistically significant', is quite small. Bradley and Taylor (1998) calculate an estimate of 0.7 percentage points (of pupils achieving 5 or more $A^{*}$-C grades at GCSE) for every 100 additional pupils.

These small gains in attainment have to be balanced against Garrett's other conclusions:

- that students are more engaged, have greater connectedness with school, and participate more fully in smaller than larger schools;

- that teachers have more positive perceptions of school climate and their ability to influence school policies, and perceive clearer norms and greater levels of co-operation;

- that virtually every study shows students from lower socio-economic backgrounds achieving better in smaller schools.

This last conclusion is to be found in many US studies, even those which are more uncertain about overall attainment. (See, among others, Berlin and Cienkus 1989; Fowler 1995; 
Friedkin and Necochea 1988; Howley 1994; Lee and Smith 1995 and 1997.) All these studies conclude that large schools have a more negative impact on ethnic minority pupils and those from lower socio-economic backgrounds. It appears that smaller schools are generally better able to mitigate the poverty effect. This is compounded by a tendency towards poorer attendance and behaviour, and the higher number of school dropouts, in larger schools.

At this point, it is impossible to disentangle school size from the structural issues referred to earlier in this report (the degree of specialisation, the large number of different teachers which each class faces during the week, the very limited time a class spends with any one teacher). It is worth noting, however, that the small school reform in the USA has involved both size and structure. Indeed, one of the key researchers Craig Howley (2004) suggests that the data would be far clearer if one could separate schools which are 'small by design' from those which are 'small by default'. Some small schools retain structures more typical of larger schools, and therefore fail to produce more cohesion - just as some teachers with small classes continue to behave in an impersonal manner. Conversely, some larger schools have been able to improve pupil-teacher relationships. Howley notes, for example, that some 'schools within schools' have very limited autonomy. Smallness does not necessarily result in different practices. In the words of John Goodlad (1984:309):

It is not impossible to have a good large school; it is simply more difficult.

The positive impact of smaller schools on the achievement of low SES and ethnic minority pupils, as well as the social benefits, have enormous implications for many parts of Britain, and especially Scotland where the concentration of poverty is great. In Scotland, a third of the school population are growing up below the poverty line. In both England and Scotland, government policy currently encourages larger schools (see, for example, the Five Year Strategy, DfES 2003). Recently in Scotland, some proposed school amalgamations have been successfully resisted by parents.

\section{How extensive are problems of social cohesion in the early years of Scottish secondary schools?}

Exclusion statistics provide a useful quantitative indicator suggesting a pattern of troubled relationships and behaviours, and of pupils finding it difficult to engage with school. There has been significant concern about this recently, with some attempts to generate a moral panic about young people's behaviour. Examining the issue from a different angle, the data can be seen as an indicator that many pupils from lower socio-economic backgrounds in particular find the early years of secondary school unsettling.

According to Scottish government statistics ${ }^{\text {iv }}$ (SEED exclusions data), there were 36,496 exclusions in the 2002-3 session, or 50 cases for every 1000 pupils. Almost all of these were temporary. (332 pupils were taken off the school's register.) Contrary to popular myth, only $4 \%$ of exclusions resulted from physical abuse of staff, and 13\% from physical abuse of other pupils. The majority are ascribed to 'persistent disobedience' or 'bad language'.

$85 \%$ of all exclusions are from secondary schools, amounting to 98 incidents per 1000 secondary pupils. The incidence rises slowly through primary school, but leaps on entry to secondary school, increasing fourfold from P7 to S1. The frequency continues to increase over the next two years, decreasing in S4, the final year of compulsory education in Scotland. Incidences are, understandably, low in the post-compulsory years. Taking S1-S3 together, there are about 125 incidents per 1000 pupils.

Exclusion is nearly four times as likely for boys as for girls. A reasonable estimate is 197 exclusions per 1000 pupils, among S1-S3 boys ${ }^{v}$.

The official data gives various other breakdowns, for example by socio-economic background, special needs, etc. but does not seek to cross-refer the various tables. Despite the hazards, it is revealing to attempt a synthesis of these figures. 
Exclusions are more frequent among pupils entitled to free school meals (FSM, the most frequently used poverty indicator in school statistics). From a general baseline of 50 per 1000, it rises to 120 per 1000 for pupils on free school meals (both sexes, all ages), or 2.4 times as high. A reasonable estimate would therefore be 473 exclusions per 1000 pupils for S1-S3 boys entitled to free meals. Similar calculations produce estimates of 429 exclusions for every 1000 S1-S3 boys with Records of Need (i.e. special educational needs above a certain level of gravity), and 894 exclusions per 1000 'looked-after' boys. ${ }^{\text {vi }}$

Data also exists on a regional breakdown. Taking the lowest and the highest, we would have to multiply by a factor of 0.14 for Shetland (i.e. reduce to about a seventh), but 1.82 for Dundee and 1.74 for Glasgow (nearly double the national average) ${ }^{\text {vii }}$. It would clearly be inappropriate to use both the area factor and poverty factor simultaneously, but legitimate to cross-match area with gender and age-range. This results in estimates for Dundee (a city with a hgh concentration of poverty) of 357 cases of exclusion for every 1000 S1-S3 boys.

It is important to note that we are dealing here with the number of events in a year, not with the number of individual pupils, since some pupils are excluded more than once. Overall, the 36,496 exclusions involved 20,180 individual pupils. Allowing for the possibility that the ratio might not be uniform across different ages, genders or social categories, an estimate can be reached by using a factor of 0.55 (20180/36496). This suggests that around 260 separate pupils are excluded in a year per 1000 among S1-S3 boys with a free meal entitlement. Such a figure is alarming: in other words, in a single year, during S1-S3, boys on free meals stand a one in four chance of exclusion from school.

This data, although not absolutely reliable, is sufficient to indicate considerable turbulence in the early years of secondary school for a significant population of pupils.

Although further comparison of data is beyond the scope of this paper, it is important to note that in Scotland, as in the rest of the UK, there is considerable inequality of learning outcomes. A recent report, drawing on data from the 2003 Scottish School Leavers' Survey, establishes that $71 \%$ of those with a parent in a higher professional or managerial occupatiion achieved five or more Standard grades 1-2 (Credit), compared to just 17\% of pupils from families in 'routine' occupations. While only $6 \%$ of leavers in the highest social class failed to achieve any Standard Grades 1-2, the figure was $48 \%$ for the lowest groups. Roughly similar data emerges for grades 1-2 in either English or mathematics. $88 \%$ of those with a parent in a higher professional or managerial occupation stay on at school after age 16, compared with $48 \%$ from families in 'routine occupations' (summary by Monro, TESS 10.12.04).

Even without a direct statistical correlation, it would seem reasonable to consider that an unsettled experience of the early years of secondary education might in part contribute to lower attainment at age 16. The Scottish Parliament has sought to improve attainment, particularly for the most disadvantaged groups, by reducing the size of English and maths classes in lower secondary school from 30 to 20 . This should have a significant impact, but if the turbulence of the lower secondary experience results partly from a sense of anonymity and disengagement which is related to school size and/or the curriculum and staffing structure, the problems will not be overcome by a reduction in class size alone.

It is time to consider the pilot study comparing a Scottish school with two Norwegian schools.

\section{The case study schools}

The schools were chosen opportunistically, because of ease of access, though advantages emerged during the study. The Scottish school, set in a relatively (though not extremely) deprived part of a major city, is of moderate size, but with the customary organisational pattern described above. It has a dedicated and caring staff. One of the Norwegian schools is situated in a moderately sized town, the other serving several villages and the surrounding area. Both are small by British standards, with less than 100 pupils in each of three school years. Like the Scottish school, the staff appear to be committed and the ethos good. There 
was no clear distinction between school buildings; indeed, one of the Norwegian schools experienced poorer facilities than the Scottish school. Nor was there any indication that any of the sample schools were atypical in terms of the extent of staff or curriculum development, for example.

\section{Methodology}

This is a mixed-method study, using three complementary approaches to compare schools in both countries:

- a small-scale questionnaire, with questions focusing on pupils' sense of personal efficacy;

- focus group discussions, designed to explore features of pupils' experience and perceptions of school, including relationships and their experience of school work;

- lesson observations, shadowing a class through a complete day.

To ensure similar ages, these methods were used with S2 classes in a Scottish school (upper and lower set) and a first year class in two Norwegian schools. Interviews and informal discussions with teachers were used to clarify aspects of school and curricular organisation.

\section{The questionnaire}

A survey was administered to one class in the Scottish school, and, in translation, to one class in each of the two Norwegian schools. We had originally intended to use this tool with the other Scottish class which we observed, but this proved impractical. Since the Scottish school was setted, and it was the higher set which responded to the survey, this might potentially place the Scottish school at an advantage, or at least compensate for lower levels of poverty in the Norwegian populations. (Norwegian classes are unstreamed.) The results were analysed in a variety of ways, using SPSS software.

A survey, based loosely on Richards and Neill's Life Effectiveness Questionnaire (LEQ), though avoiding duplicate items, put 16 statements requiring pupils to evaluate their own qualities and approaches in learning. They included questions referring to personal organisation, peer leadership, and communication skills. For example:

I am open to new ideas.

I am a good leader when a task needs to be done.

When I do a task, I am confident I will succeed.

I communicate well with adults.

Pupils were asked to respond using a five point scale:

$\sqrt{ } \sqrt{ }$ nearly always / very true

$\sqrt{ } \quad$ often / quite true

? $\quad$ sometimes true

$\mathrm{X}$ often false

XX definitely not true

A further option was available, 'I am never in this kind of situation' (0).

Overall, both Scottish and Norwegian pupils tended towards the positive side of the spectrum $(?, \sqrt{ }$ or $\sqrt{ } \sqrt{ })$ :
$1 \% 0$
$1 \% \mathrm{XX}$
$6 \% \mathrm{X}$
$25 \% ?$
$45 \% \sqrt{ }$
$23 \% \sqrt{ } \sqrt{ }$

However, major differences emerged, the Norwegian pupils' responses to almost every question being substantially more positive than the Scottish pupils. The total number of pupils answering positively ('true' or 'very true') was expressed as a percentage of the total number of respondents. The Norwegian pupils' percentages were (with one exception) consistently higher than the Scottish; combining the responses to all 16 questions, there was a difference of 20 percentage points (73\% compared with 53\%). (The only question where the Norwegian response was marginally more negative was to the statement 'I do not waste time' which only 
about a third of Scottish and also of Norwegian pupils identified with. This indicates that the Norwegian pupils were not thoughtlessly positive in their responses, and that honesty and realism are still common virtues among young people of both countries.)

There were particularly big differences in response (by 36 and 37 percentage points) to the statements:

I have the ability to do anything I want to do.

I don't get worried, even if the task is difficult.

These two statements were identified as 'statistically significant', as were:

I plan and use my time efficiently.

I am successful in social situations.

I do my best to get the details right.

I change my thinking if there's a better idea.

I try to get the best results when I do things.

The lowest levels of assent were from the Scottish pupils, to the statements:

I don't get worried, even if the task is difficult.

I am a good leader when a task needs to be done.

Many of these differences suggest that the Norwegian pupils have acquired greater selfconfidence in themselves as learners, and in their ability to operate in the school environment. Part of this may be due to higher levels of social deprivation in the Scottish school, though this was partly mitigated by the application of the questionnaire to an upper set. However, putting this evidence together with the focus group interviews and the observations summarised below, it appears more likely that it is a consequence of the way the schools are structured, and of the learning cultures which are made possible by these different forms of internal organisation.

\section{Focus group interviews}

A short (20-30 minute) semi-structured interview was held with five pupils each in both of the Scottish classes and both of the Norwegian schools. The results are summarised below. The pupils' responses should not automatically be assumed to be reliable, though even when they are not, they may still be valuable as reflecting the way the pupils understand their realities.

How well do pupils and teachers know each other?

How many teachers do you have altogether?

Do any teachers know you really well?

Do you feel confident talking to them all?

If you had a personal problem, would you discuss it wth a teacher?

To give a greater degree of shared meaning to these questions, we added as examples:

(Q2) If you have a hobby, like playing for a sports team or a musical instrument, would your teacher know that? If there was a new baby in your house, would they soon know?

(Q4) Suppose you were in trouble with the law, or your parents were arguing a lot and that was bothering you, would you feel able to discuss it with any teacher?

The Scottish pupils estimated the number of their teachers as 12 or more, the Norwegians as 5 or 6, adding, in one school, that two of them are our 'main teachers'. (This reflects a pattern whereby one or two teachers have a guidance and pastoral role for a class, as well as teaching the class for a substantial portion of the week.)

The Scottish pupils did not feel that their teachers would know such details of their lives, but there were some differences in the response to other questions. The lower set pupils said 'No, couldn't talk to them, any of them. No, I wouldn't go to anybody with a problem'. Some of the upper set pupils said they could approach their SE (PSE / Guidance) teacher; they felt 
confident to talk to teachers but 'not about personal problems, just stuff.' The Norwegian pupils answered very readily that their teachers knew them well. They all agreed on this. 'They know us. Our teachers know about our interests. They also know where most of us live.' 'Yes, our main teachers know us well. They know our hobbies'. One of the Norwegian pupils insisted he could not discuss a family problem with his teacher - they were related! One pupil explained:

We write a log which the class teacher collects at the end of the week. In that log, we can tell the teacher whether we would like a meeting with him. One of the questions we answer in the log is 'How are you?'

What is the scope for exercising choice during time at school?

When do you have a choice about what work to do in school? [We asked the pupils to give a typical situation.]

What do you choose about?

How do you decide?

How often do you have choices? [which subjects]

The Scottish pupils gave some examples of choice: 'PE sometimes... football or basketball' 'Last day of term.' 'Powerpoint.' 'In technical...candleholder.' 'Can decide trips out.' They gave the impression that these were quite rare events. Ironically, in one art class, we had just seen a wide range of choice exercised by the pupils; perhaps the pupils have come to think of school as a place where they do not exercise choice, which is interesting in itself. One added, 'The teacher says what to do.'

The Norwegian pupils were quite articulate and forthcoming in response to these questions.

Yes we do have choices in some subjects. Very often in PE. We get to choose themes for stories to write in Norwegian. We've also decided on some excursions, as part of our studies, for example to the Eidsvoll [the hall where the Norwegian constitution was drawn up, and independence was claimed].

In the middle period, we can choose what to do, for example what kind of sport we want to practice. Sometimes in some subjects (maths, Norwegian, social subjects) the teacher writes a list on the board of different tasks we can do, and we choose from the list.

Nevertheless, they felt this wasn't really enough - 'We don't make many choices in a day.' Others referred to the Storyline activity they were planning (a two-day social studies project see below), saying 'We don't think there are going to be any freeloaders. Everybody will contribute.' Project work was quite common, and one pupil explained, 'We chose our own planet in science, and when we were doing Norway, we each chose which towns we would find out about.'

How much discussion is there, and opportunities to express opinions?

How often are you able to give your own opinion and ideas?

Can you tell us when, or give us some examples?

The Scottish pupils' responses varied according to the class. The lower set seemed unable to answer this, offering us only 'Pizza' on which we could obtain no further information. The upper set gave various examples: 'We had a debate about euthanasia in RE.' 'We had a discussion in English about teenagers... sex, drugs, rocknroll, you know!' Because we were aware that sometimes Scottish teachers use 'discussion' to refer to a whole-class activity which is closely controlled by the teacher's questions, we asked them to explain how this works: 'Who speaks first? Does anybody ask questions? How do you sit?' We were told that the teacher asked different pupils questions, and the pupils were seated in rows.

Both Norwegian groups answered this question enthusiastically: 
All the time! You just have to put your hand up! We're encouraged to follow the news and very often we discuss the news in the morning. It's much more interesting to listen to the discussion if you've seen the news. Yes, we have real discussions. One pupil responds to another pupil's statement, and that person might explain more after that as well. We discuss a wide range of topics: TV programmes, the royal family, the European Union... We have most discussion in religion, Norwegian and social studies.

We give our own opinions in every class! We have discussions in the classroom every day - mostly we discuss in Norwegian, English and social studies.'

What is the extent of cooperative work and a sense of audience?

Do you work in groups?

Do you ever present or read or perform your work to others?

Working in groups was a feature of Scottish and Norwegian schools. The Scottish pupils mentioned particular subjects (French, art, PE). They referred to an activity of making a board game in business studies, and forming quiz teams for English. The Norwegian pupils were emphatic:

Yes, very often - but on Thursdays, in the middle hour ${ }^{\text {viii, }}$, we have to work alone. We've also had group tests in social studies. Some pupils prefer to work alone.

We usually sit in pairs in the classroom, but sometimes in fours. In these settings, we discuss and exchange ideas very often.

The Scottish pupils mentioned sometimes doing a talk in English, or telling the class about a film they'd seen. or doing a poster. They also mentioned putting a display on the wall in French, or having a photograph displayed in the French corridor. One group also mentioned working on a mural [with a community artist]. We had the impression that presentations were more frequent in the Norwegian classrooms, and formed the natural conclusions of group investigations.

Yes, we sometimes have presentations on our own in front of the class. We have to present all our group projects. You can have a little note in front of you, but it is better if we don't read directly from it. Sometimes we use overheads or maps to illustrate what we're saying. We also perform plays in Norwegian; the more you adapt to your role, the better it is.

In social studies ${ }^{\mathrm{ix}}$ we have to study issues in depth. We work in groups and present our findings in front of the class. Sometimes we make posters and hang them on the classroom walls.

How does differentiation of work operate?

It is important to note that setting or streaming is not practised in Norwegian schools (though one school had a small withdrawal group for literacy skills.) The Scottish school sets by subject / groups of subjects, but this does not of course eliminate differences within classes. Many pupils are together in the lower or upper set for many of their subjects. An important way in which Norwegian schools provide differentiated work is through the class's fortnightly learning plan, a statement covering the whole curriculum which details all the tasks which individuals are expected to complete. This provides alternatives for some tasks, whether more challenging or more basic, and often the pupils make the choice. Around one lesson per day is left free of formal teaching to work on the fortnight's tasks, and the rest are completed at home.

How is the work adjusted so that it is suitable for different people in the class?

Who decides what you should do? How? Do you have a say?

Give an example of different people getting different work?

Is the work usually right for you? 
The Scottish pupils in both upper and lower sets mentioned 'helpers', specifically in Maths, English or French. Apart from that, they believed that all pupils in the class do the same work. There was, in both groups, concern about mathematics. From a lower set pupil:

Maths is too easy ...they said we didn't pass it, but we did, so we're in the wrong group.

Conversely, from an upper set pupil:

If you're moved up a class, it's hard because you haven't done the work.

The Norwegian pupils gave the following accounts:

Everybody in the class is on roughly the same level. We can choose whether we want to do the harder tasks. What we are given in homework sometimes varies between the levels we are on.

The tasks are adjusted to different levels, but sometimes people who are not as clever get more help from the teacher. There's also a group for people who need extra help with Norwegian. At the end of each fortnight, we each write a self-evaluation about the learning plan and the tasks we've carried out. We say what we were satisfied with, and if we had any difficulties. We like that system.

However, this explanation was prefaced by the belief that 'We're all at the same level, so we don't need much adjustment.'

Sources of confusion (in class / around school), and how the school experience might be improved

Do you ever get confused in class?

What causes this? How do you solve it?

Does anyone help? Try to give examples.

Do you ever get worried around the school? Why?

What would improve it?

It was at this point that the Scottish pupils, in both classes, made explicit references to the degree of teacher control. With some pupils, the tone was critical, but with others, it was simply a neutral explanation, the reason why nobody's confused. Nevertheless, it marks a significant cultural difference compared with Norwegian schools. Here are some responses:

No. They say sit, you jump, you do what they say.

They tell you what to do... when you have to do something.

You find classes. You get used to it.

The Norwegians' responses were more learning-focused.

It varies from person to person... You can be confused if you're tired...If you've got a headache, then you sit by the window... We are maybe less focused on Fridays we just want to get out.

They ask the teacher or another pupil if they can repeat what has been said. When we have study hours, it alternates between 'quiet hour' and 'co-operation hour'.

The lower set Scottish pupils asserted very firmly 'No, no,' they didn't like school. They particularly wanted places to relax. Unfortunately, with the upper set pupils, time was short, and we didn't obtain a response. The Norwegians asked for 'more activities in the school yard... and to start later in the morning.' 'We want new showers in the cloakrooms.' Some referred to 'mobbing' (a near synonym to 'bullying', with public and in-school campaigns to eliminate it.)

During the winter, we sometimes get 'baptised' in the snow. It's not funny.

\section{Lesson observations}


There is not the space to give detailed descriptions of the 20 or so lessons observed, but it is useful to make some general characterisations. None of this is intended to be critical of individuals, but may help to convey some of the differences of learning culture into which teachers, as well as pupils, have been socialised and which come to appear as 'normal' within a particular education system. The specific examples given are not intended to be representative, but simply to illustrate cultural features.

\section{Scotland}

The Scottish lessons tended to be closely teacher-directed, and sometimes activities were carried out by the teacher which pupils could safely and easily have done for themselves. A strong use was made of demonstrations, and lessons were often carefully structured. Pupils were explicitly held back from taking initiatives in doing valuable activities on several occasions (for example, using magnifying glasses to study crystals). In another class, the pupils made various suggestions on how they might investigate something, but were always told 'next time'. (One pupil commented loudly, 'It's always next time.') Very little of the pupils' work could be described as 'exploratory'; immediate answers were expected to teachers' questions, rather than attempts to investigate or research. By contrast, in the art lesson, pupils were encouraged to make choices, including the medium they were working in. The communications were much more fluid and multi-directional in this lesson, and pupils' work was celebrated through display. In this subject pupils seemed comfortable exercising initiative.

The teachers generally focused well on the pupils, seeking to relate to them positively and give clear explanations pitched to the right level. All teachers seemed to be positive about pupils, although there were signs of strain in this, including pupils being thrown out of lessons, or others being denied entry. Pupils were generally well behaved, and listened well, but often not really engaged.

The standard furniture arrangement was in rows, but there were round tables in the library and, in one room, computers round the walls. It was surprising to see a class of two pupils (a reading recovery lesson) seated in a small classroom at desks similarly arranged in rows; there were only about 12 seats in this small room altogether. Even in this class, the normal style was of synchronous activities, and when either of the two pupils tried to expand or develop an idea, the teacher quickly moved them on.

Resources were good (much better than in one of the Norwegian schools). There were interactive whiteboards, and well written and illustrated text books. However, pupils were rarely given time to use the textbooks. For example, only 20 or 30 seconds was allowed to study a page about the structure of the earth, and to make sense of the illustration and its labels. The teacher soon substituted his own explanation. Perhaps the assumption was that the pupils' reading skills were too weak to make sense of this; the problem, however, is that the reading does not develop.

The teachers frequently asked questions, and there was little pupil contribution except in answer to specific questions. Pupil contributions lasting more than three or four words were rare, and hardly ever legitimised:

- In the lower set, two pupils gave longer answers but the teacher spoke across them.

- In an upper class, pupils made various attempts to engage in discussion of the topic, but the teacher didn't seem to notice the raised hands.

- Another teacher simply talked over pupils if they began to develop a point beyond a specific two or three word answer to the teacher's question.

Occasionally, the pupils would answer in chorus, and the teacher was able, by his reaction, to turn yes into no, or a mixture of yes and no into a universal yes. 
Even in the upper set, there were, from some teachers, overt signs of limited expectations: 'I know writing's not your strong point.' There was rarely any attempt to find out what pupils already knew; at other times, this was done by asking the whole class a question, and a response from one or two pupils was taken to mean that the class as a whole would know.

Higher levels of thinking were quite rare. There were some glimpses of this in some pupils' responses, but no attempt to build upon them or to develop a deeper understanding through sustained dialogue. There was a tendency for writing to consist of copying or answering straightforward factual questions. Often a small adjustment to a task would have forced the pupils to think rather than simply carry out a task as routine. There was little sustained discussion in which pupils could handle and develop more complex ideas. In some lessons, the pupils might have benefitted from more direct experience or objects or models to handle.

There were strong boundaries between subjects, and sometimes confusion. For example, they had been studying the earth and solar system in both geography and science, but without any liaison, the teacher was left saying, vaguely, 'You should have done it in geography?'

There were signs of a clear difference of expectation between the upper and lower set. In the lower one, the emphasis was 'you can't expect too much of this class' (said within the hearing of pupils) and 'you have a bit of fun - not too much focus on tests'. The upper set, although only S2, were being reminded constantly of the examinations at the end of S4: 'It's important to learn this, it'll come up in your Standard Grade.'

It is worth mentioning, as a possible indication of cultural differences between education sytems, a fortuitous incident involving a pupil who had one Danish and one Scottish parent, and who had recently moved to Scotland. (This is non-judgemental, and we have no other knowledge of the pupil's behaviour or past record.) She made it clear that she resented and resisted the norms of classroom discipline in the Scottish school. She commented, 'Scottish schools are very strict ... there are always punishments. You never get punished for talking back to teachers in Denmark.'

\section{Norway}

Many lessons in Norway were conducted as whole-class lessons, and co-ordinated by the teacher. The word 'co-ordinated' is perhaps the best word to describe a style in which pupils freely take part in a kind of open conversation. We saw relatively little direct instruction, maybe because we were approaching the end of the school year. (Perhaps there is too little direct teaching.) The curriculum appears to be built less out of relatively self-contained lessons, and more on longer term activities of which we saw various stages - an announcement of a forthcoming project; a discussion of what questions they could ask on tomorrow's visit; pupils presenting their findings to the class; evaluations of tasks and tests. One class was going to visit a nearby lake, and had been introduced to ph tests. What questions did they plan to ask? A teacher presented statistics showing how the class had done on a test, explained certain answers, and pointed out common problems and invited discussion of them. In addition to our day of lesson observation in each school, we observed a Maths Day, in which groups of pupils had designed activities which others then toured.

One class were introduced to the Storyline they were to begin in a few days. This would be shared with other classes in the same year. Storyline is a particular kind of thematic work built around a narrative structure. It is popular in Scandinavia, where it is known as 'the Scottish method'. (This came as quite a surprise: although invented at Jordanhill teaching college, it has been largely squeezed out by a national curriculum built from distinct subjects, and perhaps also by accountability pressures.)

You will spend a whole day and two half days on this. We will divide into two communities, based on our two villages. On the first day, you don't need to come into school: you can meet up in small groups anywhere in the two villages, but you must 
text us at 9 and at 2 to say where you are. It's a kind of role play, where you will divide into families of four people. It's up to you how to organise your family. [Pupils suggest single parents, grandparents, a gay partnership.] Yes, it's entirely up to you, except that everybody should be at least ten years old. Your first task will be to write a card which presents the character you are: work, personal interests, position in the family, income and so on. You will have to find out more about the kind of life you're living and how this fits in to life in the community. Your teachers will have particular roles - postmen, police officers, church ministers - and from time to time they will make things happen. There will also be public discussions and you'll have to act in character and stand up for your opinions and interests. In the end, you'll present your activity on posters.

The pupils were clearly accustomed to organising themselves in such extended activities, and the teachers felt they would be well motivated and could be trusted. The teachers were respected, but relationships were quite relaxed and good-humoured. Teachers expressed confidence about pupils' abilities:

I know you know this. Still, it's easy to forget so we'll just make sure.

[One boy asks if he can be an infant, in the Storyline.] Yes, you often play that part, but not this time. Why not try something else?

There was no set pattern to furniture, which was moved around during the day. However, unlike the Scottish school, the classes spent most of the day in the one room. One classroom was poorly furnished, with quite old desks with vertical boards attached; pupils organised these in a haphazard fashion to make study carrels, giving themselves a degree of privacy in study lessons. There were no whiteboards, though textbooks were good. The science equipment in one Norwegian school seemed poor, compared to the Scottish school, but did have the advantage that it could be used safely and flexibly by pupils with minimal supervision. (Instead of gas taps in the science room, pupils borrowed small camping stoves from the storeroom.) On the field behind one school, we saw a three-sided hut which the pupils had built, open at one side and large enough to shelter a class from snow and rain. This was used for a variety of learning and recreational purposes, including outdoor science lessons.

Patterns of communication were of particular interest. Open questions were frequent: examples.

What are the various features of a short story? Would you like to give some

or the homework question:

Ask an adult about a political crisis they remember, and what caused it.

Higher level questions and tasks were frequent in the textbooks used in independent study periods. The pupils themselves often asked questions. Presentations to the rest of the class were a normal conclusion to investigations and projects.

We began to notice a pattern in whole-class communications. In Scotland, as in some other English-speaking countries, interactions are generally controlled by the teacher's question (in the IRF or Initiate / Respond / Feedback pattern described by Sinclair and Coulthard (1974); the teacher initiates, a pupil responds, the teacher gives feedback, perhaps simply repeating the response, before opening another IRF sequence). In our Norwegian classrooms, the teacher would tend to begin to speak quite slowly. After two or three sentences, s/he would pause for a few seconds. A pupil would make a contribution. The teacher would then engage for several exchanges in a dialogue with that pupil, or others might join in. Alternatively, even before the pause, a pupil might interrupt the teacher, even finishing off the sentence.

Another interesting variant on question and answer came in the form of a quiz. The groundrules were different to those one might expect. As well as answering questions in this 
news quiz, pupils would freely comment on recent vents. The pupils explained that they saw it as an incentive to watch the television news; it did not function as a test.

Another interesting aspect was the use of questions not to trigger existing knowledge but to encourage further enquiry. This was a use made by teachers, but could also characterise how teachers responded to pupils' questions. Before the trip to the lake, one pupil asked, 'So if the water is sour, what happens to the fish?' The teacher withheld an answer, inviting the class to research this further.

Sometimes the cognitive level of an activity was low. Quite a long time was spent making models of planets, for example, with no apparent direction or discussion. (Perhaps that came before or after, and it was judged important to give time for sensory experience.) Frequently however pupils were engaged in challenging exchanges with teachers or each other. The textbook questions for independent study were pitched at a high level, and pupils frequently wrote a half or whole page in response. Some examples:

- Why does the climate in Norway vary so much from place to place?

- Why are Norwegian rocks sometimes found in Denmark?

- What makes good soil for agriculture?

- What were the consequences of specialisation in occupations?

- Study the painting 'Strike'. How does the painter use light? What do you think are his intentions?

Pupils were generally well behaved, and the atmosphere relaxed. Several times we saw friendly banter between pupils and teachers. On one occasion pupils locked the teacher out of the class; his response was serious but not over-excited: 'Jokes like this can be very funny, but I'm not feeling amused by this.' The lesson soon continued. Frequently pupils worked without overt supervision, or even without a teacher in sight. Pupils were expected to take responsibility for their own learning:

P: I don't think I have that in my book. I was probably ill that day.

T: You know that it's your responsibility to copy from your fellow pupils if you've been away. Make sure you copy it in the break now, so that you have it for your test tomorrow.

Tests were quite frequent, and were taken seriously, both as a check at the end of a fortnight's learning plan and at year end. The latter were often open-book tests, with a text to prepare beforehand; they tended to consist of one or more extended task, rather than short recall answers or essays. One teacher congratulated some pupils for spending three hours on their test; another reminded a class to bring enough food and to take proper breaks. [They were allowed to leave the room when they needed to.] Other end of year tests were oral: make a presentation on [depending on the subject] the Cold War; genes; disease prevention; triangles. Or in English, after studying a textbook and internet sites, to speak about the American Declaration of Independence, including the revolutionary war and women's situation at the time.

\section{Conclusions}

It is frequently difficult, in education, to establish a direct causality, since so many factors are at play. This study is no exception. Should we ascribe the patterns of classroom learning to the teachers' personalities; to their training; to the national traditions; to the official curriculum documents; to historical features of the education system; to school size; or to the organisation of the curriculum and school? Any or all of these could be a factor. As we saw earlier, the extensive American research on school size and associated structures has not shown an automatic effect on achievement, but there is sufficient regularity to indicate a formative influence. Recalling Goodlad's formulation once more (1984:309):

It is not impossible to have a good large school; it is simply more difficult. 
Similarly, it would be going too far to suggest that the learning cultures we found in Norwegian schools were a direct consequence of the year team organisation, but, examining the three sets of data (from the questionnaire, the focus groups and the observations), it seems fair to argue that the structure generates sufficient trust for such a pedagogy and learning culture to flourish. Of course, individual subject teachers can do this in the tighter frame of the Scottish curriculum but it is much more difficult and requires unusual talents and determination. When a teacher only sees a class for an hour or three a week, it seems much safer to take a more controlling stance. The teacher might realise that sometimes it would be good if a pupil could interrupt with an idea, or if the chain of contributions to a discussion could move round the class, or if the pupils could start asking questions, but this is dangerous if you're not too sure about the individuals and you could lose control of the class; safer to frame their contributions in a more disciplined way through your succession of closed questions. To move outside such patterns really does require a closer knowledge of your class than you have time for, and a well established nexus of trust. Besides, if you're worried about 'covering the syllabus', you need to maximise the transmission of knowledge rather than allowing for exploration and discussion.

We are dealing inevitably with tendencies and favourable conditions, rather than direct causality, but conditions and structures are important when considering educational change. To paraphrase Brecht's Galileo, 'Unhappy the school that needs heroes.'

We sit at a particular crossroads in the development of schooling. It is not hard to see that 'kids are different today', and that their expectations and experiences are often out of step with the established patterns of schooling. This is not to suggest that schools should simply assimilate to youth cultures, but they must adapt to changing circumstances. Nor are the learning needs the same as they were; the role of teachers in transmitting knowledge has become less central in the computer age. The world is awash with easily accessible information, but it is all the more important for young people to learn how to select and critically evaluate it. The learning culture of the Norwegian classrooms does seem more in tune with these changing conditions and expectations.

Scottish teachers are constantly being asked to transform their practices - to differentiate, to be more inclusive, to improve the school ethos, to develop creativity and critical thinking, to make schools more conducive to democratic citizenship, as well as raising attainment. Such changes certainly need collegiality, time for reflection and well-planned staff development, but even with all these, it is difficult to see them succeeding within the present highlyspecialised structures where, at best, the class-teacher contact amounts to three hours a week.

And this is before we even consider the role of schooling to socialise young people, to promote their social and moral and cultural development, to foster empathy and a sense of justice. These also take time, and cannot be relegated to the PSHE lesson.

Most young people survive and gain qualifications and turn out to be reasonably civilised human beings in today's schools, but do they really prosper? For many others, the experience of schooling is unsatisfying and even parlous. A situation in which a quarter of the 12-15 year old males of Dundee or Glasgow, or of adolescent boys on free meals or with Records of Need, suffer exclusion from school each year must give cause for concern. For some pupils, an exclusion will serve as a warning to change their ways; for many it will further promote their sense of disengagement from school and all it stands for. It cannot be called 'inclusion'.

School exclusions do not lead directly or inevitably to alienation in later adolescence, or antisocial behaviour, or trouble with the law, but sometimes they do, and even if this were never to happen, it is hard to describe our present schools as well developed nurturing communities. They can be unsettling even for well adjusted young people, let alone for those whose lives are already troubled.

The challenge which Scotland's inspectors identified nearly ten years ago (SOEID 1997), and then ducked, has not gone away in the meantime. The problem cannot be overcome by 
school-level initiatives alone. It would require an unfreezing of current patterns of teacher education, and a more flexible approach to teacher registration. An alternative needs to be opened to the current norm of secondary teachers registered to teach a single subject to year S1-6 (ages 12-18) ${ }^{\mathrm{x}}$. A workable alternative would be a specialism to age 18 (i.e. university entrance), accompanied by a more general qualification for S1-2, for example sciences and maths; or English and social subjects (history, geography, modern studies). Some use could also be made of the Chartered Teacher qualification to extend an experienced teacher's range beyond a single specialism. Clearly solutions cannot simply be transferred from one national context to another. However, a reform of the first stage of secondary education, in the direction of greater social cohesion, is arguably a precondition for the future development of Scottish schools and so that the transformations identified by so many progressive policy documents can become reality.

The author can be contacted by email: terry.wrigley@ed.ac.uk

\footnotetext{
${ }^{\text {i }}$ The word 'pupil' is used in this article, rather than student, as this is the normal term for learners of any age in both Scottish and Norwegian schools.

${ }^{\text {ii }}$ Guidance teachers undertake pastoral care of various kinds, liaise with parents, provide personal and academic guidance, and teach Personal Social and Health Education. Learning support teams work alongside, or together in the same team as, behaviour support staff. The management team of a typical Scottish secondary school consists of the headteacher and three or four others.

iii It has its origins in the 'grammar schools' which provided an academically oriented curriculum for around $20 \%$ of the population. Such schools were generally quite small, however, typically with about 30 teachers and 500 or 600 pupils. As comprehensive schools were established, this structure was adopted by the new, and considerably larger, schools. Moreover, the new schools served a very different, and sometimes more troubled, population.

iv Some of this data is extrapolated from graphs, accounting for approximations.

${ }^{\mathrm{v}}$ Overall, in S1-S3, 79\% of exclusions are of boys, compared with (an assumption of) $50 \%$ of boys in these year groups overall. Therefore, to estimate the number of male exclusions for every $1000 \mathrm{~S} 1$-S3 boys, we can multiply 125 (exclusions per 1000 in S1-3, boys and girls together) by a factor of 1.58 (79/50), or 197 male exclusions per $1000 \mathrm{~S} 1-\mathrm{S} 3$ boys.

${ }^{\text {vi }}$ Combining boys and girls, across all types of school, there are 50 exclusions per 1000 pupils; restricting to pupils entitled to free school meals this increases to 120 cases per 1000. Assuming that there is little difference at lower secondary level than the overall proportion, one can reasonably multiply the 197 cases per 1000 among S1-S3 boys by a factor of $2.4(120 / 50)$, i.e. 473 cases for every 1000 S1-S3 boys with free school meal entitlement. Similarly there are 109 exclusions per 1000 pupils, among pupils with records of need, compared with 50 across the whole school population. Thus, $109 / 50$ gives a factor of 2.18, leading to an estimate of 429 exclusions for every 1000 S1-S3 boys with Records of Need (i.e. special educational needs above a certain level of gravity). Likewise 227 exclusions per 1000 pupils, among looked-after children, again compared with 50 across the whole school population, gives a factor of 4.54 (227/50) or 894 exclusions for 1000 'looked-after' boys. vii 7,91 and 87 per 1000 respectively, compared with 50 per 1000 across the whole school population. This produces a calculation of 7/50 or a factor 0.14 for Shetland, $91 / 50$ or a factor of 1.82 for Dundee, and $87 / 50$ or a factor of 1.74 for Glasgow.

viii The 'middle hour' is a reference to the period set aside for supervised study rather than teacher-led instruction. The pupils work at any tasks in the class's weekly or fortnightly study plan. These plans often give some alternatives, e.g. extension tasks or simplified versions. Pupils are not restricted to tasks within the range of subject specialisms of the supervising teacher, but if they do, they are able to ask for help.

ix Social studies is regarded as a single subject, combining what Scottish schools often call the 'social subjects', i.e. history, geography and modern studies. Sometimes the emphasis is on one of these, sometimes they combine.

${ }^{\mathrm{x}}$ There are some limited alternatives to this at present, but mainly restricted to registration for a further foreign language, or to extend from a single science to the others, and some history teachers are also registered for modern studies.
} 


\section{References}

Berlin B. and Cienkus R. (1989) Size: the ultimate educational issue. Education and Urban Society 21/2 (Feb 1989), pp228-231

Bradley S and Taylor J (1998) The effect of school size on exam performance in secondary schools. Oxford Bulletin of Economics and Statistics 60, pp291-324

Cotton K. (1996) School size, school climate, and student performance. NW Regional

Educational Laboratory. (updated version accessed at www.nwrel.org/scpd/sirs/10/c020.html) Delamont S. and Galton M. (1986) Inside the secondary classroom. London: Routledge and Kegan Paul

DfES (2004) Five year strategy for children and learners. Norwich: TSO

Flecknoe M. (2003) Why do parents choose small schools? a review of the literature.

Improving Schools 6(3), pp 45-60

Fowler W. (1995) School size and student outcomes. Advances in Educational Productivity

5, pages 3-26

Friedkin N. and Necochea J. (1998) School system size and performance: a contingency

perspective. Educational Evaluation and Policy Analysis 10/3, pp237-249

Galton M., Comber C., and Pell T. (2002) The consequences of transfer for pupils: attitudes

and attainment. In Hargreaves L. and Galton M. (eds.) Transfer from the primary classroom20 years on. London: RoutledgeFalmer

Galton M., Gray J., and Rudduck J. (1999) The impact of school transitions and transfers on pupil progress and attainment. London: DfEE (Research report RR131)

Galton M. and Willcocks J. eds (1983) Moving from the primary classroom. London:

Routledge and Kegan Paul

Garrett Z. et al (2004) Secondary school size: a systematic review. London: EPPI-Centre, Institute of Education

(eppi.ioe.ac.uk/EPPIWebContent/reel/review_groups/School_size/s_s_rv1.doc)

Goodlad J (1984) A place called school: prospects for the future. New York: McGraw-Hill

Howley C (1994) The academic effectiveness of small-scale schooling (an update). ERIC

Digest. Charleston, WV: Clearinghouse on Rural Education and Small Schools, June 1994 (ED 372 897)

Howley C (2004) Small by design: critiquing the urban salvation of 'small schools'. (Paper in the symposium School structure: the rural-urban divide, International Society for Educational Planning, 9 Oct 2004)

Hargreaves A. (1994) Changing Teachers, Changing Times. London, Cassell

Hughes C. (2004) New times? New learners? New voices? Towards a contemporary social theory of learning. (Review Essay) British Journal of Sociology of Education, 25/3, pp 395408

Kahne J., Sporte S., and Easton J. (2005) Creating small schools in Chicago: an early look at implementation and impact. Improving Schools, 8/1

Lee V. and Smith J. (1995) Effects of high school restructuring and size on early gains in achievement and learning. Sociology of Education 68, pp241-270.

Lee V. and Smith J. (1997) High school size: which works best and for whom? Educational

Evaluation and Policy Analysis 19, pp205-227.

Monro N, Class gap 'remains as wide as ever', in TESS (Times Educational Supplement, Scotland) 10.12 .04

Osborn M et al (2003) A world of difference? Comparing learners across Europe.

Maidenhead: Open University Press

SEED (2004) Exclusions from schools 2002-03. Edinburgh: Scottish Executive National Statistics. www.scotland.gov.uk/stats/bulletins/00321-00.asp

Sinclair J. and Coulthard R. (1974) Towards an analysis of discourse. Oxford: Oxford University Press.

Sissing H. and Weeber F. (2001) Scholen over 'Minder handen voor de klas'. Amersfoort: CPS 
Sissing H. and Weeber F. (2002) Politiek, wetenschap en onderwijsorganisaties over 'Minder handen voor de klas'. Amersfoort: CPS

SOEID (1997) Achieving success in S1/S2 - a report on the review of provision in S1/S2 by HM Inspectors of schools. Edinburgh: Scottish Office

Wrigley T. (2000) The power to learn. Stoke-on-Trent: Trentham

Wrigley T. (2003) Schools of hope. Stoke-on-Trent: Trentham 